\title{
Chitinase-3-like protein 1 as a predictor for the progression or regression of liver fibrosis
}

\author{
Biaoyang Lin ${ }^{1,2,3}$, Shengjun $\mathrm{Wu}^{4}$, Yunhua Liu ${ }^{5}$, Longgen Liu' ${ }^{6}$, Saadiya Mushtaq \\ ZZhejiang-California International Nanosystems Institute (ZCNI) Proprium Research Center, Zhejiang University, Hangzhou \\ 310058, Zhejiang Province, China. \\ ${ }^{2}$ Collaborative Innovation Center for Diagnosis and Treatment of Infectious Diseases, The First Affiliated Hospital, School of \\ Medicine, Zhejiang University, Hangzhou 310058, Zhejiang Province, China. \\ ${ }^{3}$ University of Washington School of Medicine, Seattle, WA 98195, USA. \\ ${ }^{4}$ Sir Run Run Shaw Hospital, School of Medicine, Zhejiang University, Hangzhou 310020, Zhejiang Province, China. \\ ${ }^{5}$ Department of Liver Diseases, The Second Hospital of Yunnan Province, Kunming 650000, Yunnan Province, China. \\ ${ }^{6}$ The Third People's Hospital hospital of Changzhou, Changzhou 213001, Jiangsu Province, China. \\ 7 Virology Department, Armed Forces Institute of Pathology, Block E-16, New Cob Lane, Javed Sultan Shaheed Colony, Rawalpindi \\ Cantt, Rawalpindi, Pakistan.
}

Correspondence to: Dr. Biaoyang Lin, Zhejiang-California International Nanosystems Institute (ZCNI) Proprium Research Center, Zhejiang University, Hangzhou 310058, Zhejiang Province, China. E-mail: biaoylin@gmail.com

How to cite this article: Lin B, Wu S, Liu Y, Liu L, Saadiya M. Chitinase 3-like protein 1 as a predictor for the progression or regression of liver fibrosis. Hepatoma Res 2018;4:48. http://dx.doi.org/10.20517/2394-5079.2018.19

Received: 12 Mar 2018 First Decision: 22 Jun 2018 Revised: 18 Jul 2018 Accepted: 22 Jul 2018 Published: 17 Aug 2018

Science Editor: Guang-Wen Cao Copy Editor: Huan-Liang Wu Production Editor: Cai-Hong Wang

\begin{abstract}
Liver fibrosis is a wound-healing response of liver cells to chronic injuries caused by viral infections, including hepatitis B virus (HBV), hepatitis C virus ( $\mathrm{HCV}$ ), toxins, and alcohol abuse. The ability to stage diseases for treatment naïve patients to initiate proper medical procedures and predict the clinical causes of the disease or the treatment response is important given the increased prevalence of liver fibrosis caused by HBV, HCV and fatty liver diseases. CHI3L1 (chitinase-3-like protein 1, also known as YKL-40), which belongs to the chitinase family but lacks chitinolytic activity and is highly expressed in the liver, seems to fulfill this role. CHI3L1 is a non-invasive staging marker for liver fibrosis caused by HBV, HCV and non-alcoholic fatty liver disease as well as a predictor of the clinical causes and fibrotic changes after treatments. CHI3L1 predicts histological progression of liver fibrosis and fibrosis progression rate (fibrosis unit/year), rapid fibrosis progression after liver transplantation and response to interferon and recent direct acting antiviral therapy in chronic HCV patients. CHI3L1 also predicts response to antiviral therapy in chronic HBV patients.
\end{abstract}

Keywords: CHI3L1, liver fibrosis, progression, regression, hepatitis B virus, hepatitis C virus, treatment response

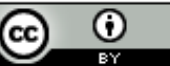

(C) The Author(s) 2018. Open Access This article is licensed under a Creative Commons Attribution 4.0 International License (https://creativecommons.org/licenses/by/4.0/), which permits unrestricted use, sharing, adaptation, distribution and reproduction in any medium or format, for any purpose, even commercially, as long as you give appropriate credit to the original author(s) and the source, provide a link to the Creative Commons license, and indicate if changes were made. 


\section{INTRODUCTION}

Liver fibrosis is a wound-healing response of liver cells to chronic injuries caused by viral infections, toxins, alcohol abuse and other causes. Liver fibrosis is accompanied by a constant process of destruction and repair of the hepatic parenchyma that is caused by inflammation and often results in serious complications, including portal hypertension and liver failure. Liver fibrosis can also give rise to hepatocellular carcinoma. Liver fibrosis can lead to cirrhosis, which is defined as the end stage of liver fibrosis ${ }^{[1]}$. In China, hepatitis B is the major cause of inflammation leading to liver fibrosis and cirrhosis ${ }^{[2,3]}$. Cirrhosis is an important factor in the development of hepatocellular carcinoma (HCC) because the cumulative 5-year risk of developing HCC in patients with cirrhosis ranges from $5 \%$ to $30 \%$, depending on several factors, including the presence and stage of underlying liver disease, ethnicity, age, sex and the duration of exposure to primary hepatotropic viruses. To reduce the burden of the end stage liver diseases (cirrhosis and HCC), it is critical to identify liver fibrosis at its early stage, predict the direction and speed of the progression, and finally to monitor and predict the treatments responses (antiviral or anti-fibrotic treatments).

Although many biomarkers (e.g., APRI, FIB4, fibrometer, fibrotest, etc.) and imaging methods (e.g., Fibroscan, ARFI, MRE) have been widely proposed for staging liver fibrosis, their abilities in predicting liver fibrosis progression are very limited. Given that fibrosis is a very slow process, it often takes years to progress or recede from one pathological stage to the next. Therefore, a biomarker that can fulfill this role is most desirable. A search for such a biomarker would require an understanding of the mechanism of liver fibrosis and the key molecules involved in the process.

CHI3L1 (also known as YKL-40) belongs to the chitinase family but lacks chitinolytic activity, which is highly enriched in the liver ${ }^{[4]}$. CHI3L1 acts as a growth factor for fibroblasts and is involved in matrix remodeling ${ }^{[5]}$. Serum CHI3L1 levels are associated with the severity of liver fibrosis caused by non-alcoholic fatty liver disease ${ }^{[6]}$, schistosomiasis ${ }^{[7,8]}$, hepatitis $\mathrm{C}$ virus $(\mathrm{HCV})^{[9,10]}$ and hepatitis B virus $(\mathrm{HBV})^{[1]]}$.

\section{CHI3L1 PREDICTS HISTOLOGICAL PROGRESSION OF LIVER FIBROSIS IN CHRONIC HCV PATIENTS}

Fontana et al. ${ }^{[12]}$ analyzed the association of serum fibrosis marker levels with the risk of clinical and histological disease progression in a large cohort of patients with chronic hepatitis C consisting of 462 prior non-responders to peg-interferon and ribavirin enrolled in the randomized phase of the Hepatitis $\mathrm{C}$ Antiviral Long-term Treatment against Cirrhosis (HALT-C) trial. They performed pretreatment liver biopsy and follow-up biopsies at years 2 and 4 and defined histological progression as a $\geq 2$-point increase in the Ishak fibrosis score in patients without cirrhosis. Clinical outcomes included development of decompensation, hepatocellular cancer, death or an increase in the Child-Turcotte-Pugh score to $\geq 7$. They collected and compared serial YKL-40 levels in patients who progressed clinically to the levels in patients who did not progress using random effects modeling. YKL-40 levels increased in both groups of patients over time $(P=0.0026)$ and were significantly increased in the progressors $(P<0.0001)$.

\section{CHI3L1 PREDICTS RESPONSE TO INTERFERON THERAPY IN CHRONIC HCV PATIENTS}

Saitou et al. ${ }^{[10]}$ analyzed noninvasive markers as predictors of interferon responses with HCV-associated diseases. A total of 109 patients with HCV-associated liver disease were enrolled, and 88 patients underwent liver biopsy. In total, 67 of 109 patients received interferon therapy. YKL-40 was superior to other fibrosis markers for predicting severe fibrosis (F2-F4) from mild fibrosis (Fo-F1) (YKL-40, AUC = 0.809; HA, AUC = 0.805). They also evaluated the changes of the levels of fibrosis markers before and after interferon (IFN) therapy. After IFN therapy, only the concentration of serum YKL-40 significantly decreased in the responder group and the non-responder group $(P=0.03)$. No changes were noted among type IV collagen, amino-terminal peptide 
of type III procollagen, hyaluronic acid (HA). They concluded that YKL-40 might be a useful non-invasive serum marker to evaluate the efficacy of IFN therapies in patients with HCV-associated liver disease.

\section{CHI3L1 PREDICTS RESPONSE TO ANTIVIRAL THERAPY IN CHRONIC HBV PATIENTS}

Wang et al ${ }^{[13]}$ compared serum CHI3L1 levels with liver tissue collagen proportionate area (CPA) and liver stiffness measurement (LSM) in a cohort of 131 CHB patients before treatment and after receiving entecavirbased antiviral therapy for 78 weeks. Before treatment, correlation analysis revealed positive correlations between CHI3L1 levels and the CPA $(r=0.351, P<0.001)$ and between CHI3L1 and LSM $(r=0.412, P<0.001)$. After 78 weeks of treatment, serum CHI3L1 levels decreased compared with baseline $(87.8 v s .69 .6 \mathrm{ng} / \mathrm{mL}$, $P<0.001)$. Furthermore, the changes in CHI3L1 are correlated with changes in CPA $(r=0.366, P<0.001)$ and the changes in LSM $(r=0.438, P<0.001)$ before and after antiviral treatments. They concluded that $\mathrm{CHI} 3 \mathrm{~L} 1$ is a useful non-invasive marker for the assessment of liver fibrosis in CHB patients before treatment and a potential useful marker for monitoring the change in liver fibrosis during therapy. More interestingly, in many cases, $\mathrm{CHI} 3 \mathrm{~L} 1$ concentrations decreased after 78 weeks of antiviral therapies, whereas histological stages based on biopsy did not change. However, upon closer examination of the histological images, they found that many samples exhibited improvement in fibrosis as demonstrated by thinning of the septa and reduction in the numbers of the septa. However, the Ishak histological stage remains the same based on the classification standards (personal communication).

\section{CHI3L1 PREDICTS FIBROSIS PROGRESSION RATE (FIBROSIS UNIT/YEAR) IN CHRONIC HCV PATIENTS}

Kamal et al. ${ }^{[7]}$ conducted serial liver biopsies in a 10-year longitudinal cohort study consisting of patients with HCV alone or HCV and schistosomiasis. Two liver biopsies were performed for patients at the time of acute HCV infection and at the end of the follow-up to calculate the fibrosis progression rate/year. In addition, CHI3L1 serum concentrations were measured yearly and at the end of the follow-up. The serum CHI3L1 change rate (difference between baseline and follow-up values) was compared with the fibrosis progression rate/year. Kamal et al. ${ }^{[7]}$ reported that the CHI3L1 change rate had a very high linear correlation with the fibrosis progression rate/year $(r=0.892, P<0.001)$. Furthermore, the CHI3L1 increase rate increases from years 4 to 8 compared with years 1 to 4 for $\mathrm{HCV}$ mono-infected patients, and the increase was noted at year 2 instead of at year 4 in HCV and schistosomiasis co-infected patients. Using data from the table of Kamal et al. ${ }^{[7]}$, we generated a scatter plot of CHI3L1 concentration and the fibrosis progression rate per year (increase in histological stages per year) [Figure 1]. As noted, no fibrosis progression is noted when the $\mathrm{CHI} 3 \mathrm{~L} 1$ concentration is $53 \mathrm{ng} / \mathrm{mL}$. As the $\mathrm{CH} 3 \mathrm{~L} 1$ concentration increases, the speed of fibrosis progression increases. When the $\mathrm{CHI} 3 \mathrm{~L} 1$ concentration is $110 \mathrm{ng} / \mathrm{mL}$, the speed of fibrosis progression is at 0.8 histological stages per year [Figure 1].

\section{CHI3L1 PREDICTS RAPID FIBROSIS PROGRESSION AFTER LIVER TRANSPLANTATION FOR HCV PATIENTS}

Pungpapong et al ${ }^{[14]}$ obtained serum and liver biopsy samples from 46 liver transplantation (LT) recipients at two time points: time point 1 , means of $5 \pm 2$ (biopsy 1) months; time point 2, means of $39 \pm 6$ (biopsy 2) months post-LT. Rapid fibrosis progression (RFP) was defined as an increase in the fibrosis score $\geq 2$ from biopsy 1 to biopsy 2 (a mean interval of $33 \pm 6$ months). They analyzed the ability of parameters, including serum CHI3L1 and hyaluronic acid (HA), histological assessment, and hepatic stellate cell activity (HSCA) at biopsy 1, to predict RFP. They found that serum HA and YKL-40 performed significantly better than conventional parameters and HSCA in predicting RFP post-LT. Furthermore, CHI3L1 (cutoff $\geq 200 \mu \mathrm{g} / \mathrm{L}$ ) exhibited $96 \%$ accuracy and performed better than serum HA (cutoff $\geq 90 \mu \mathrm{g} / \mathrm{L}$ ) in predicting RFP at biopsy 1 with $80 \%$ accuracy. 


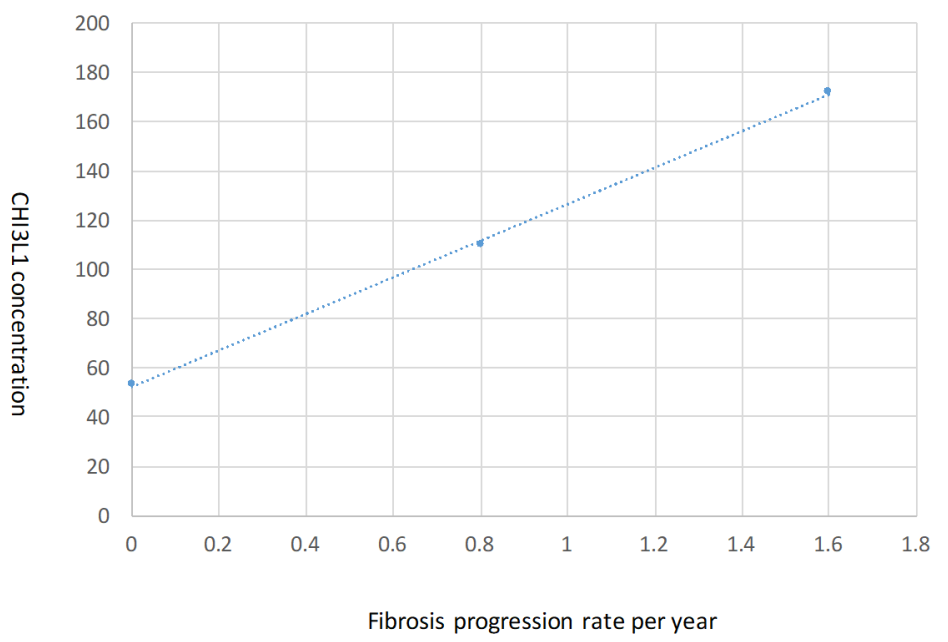

Figure 1. Scatter plot demonstrating the slope of CHI3L1 concentration and fibrosis progression rate per year

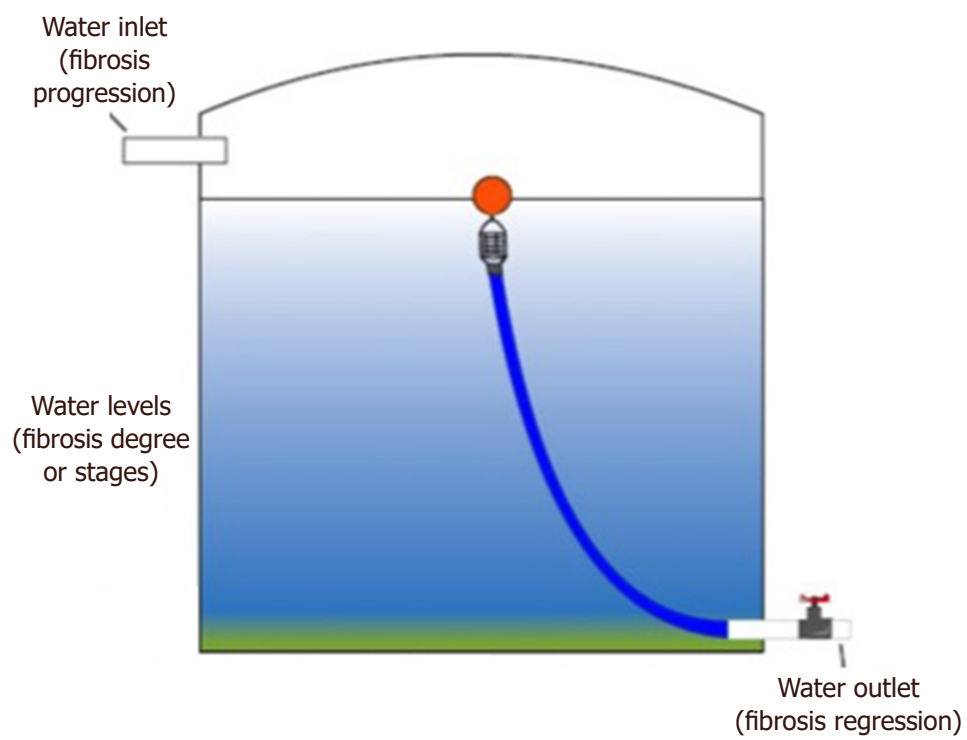

Figure 2. A water tank model to describe the relationship between the progression or regression of liver fibrosis and CHI3L1

\section{CONCLUSION}

CHI3L1 is not only a staging marker for fibrosis in treatment naïve HBV- or HCV-infected patients and NAFLD patients. CHI3L1 is also predictive of progression or regression of fibrosis. These abilities are likely due to the fact that CHI3L1 is actively involved in the process of liver fibrosis. Johansen et al. ${ }^{[15]}$ used immunohistochemical analysis to demonstrate that CHI3L1 is expressed in areas with fibrosis, particularly leading edges/areas with active fibrogenesis. CHI3L1 staining was not observed in hepatocytes but was expressed in Kupffer cells ${ }^{[6]}$ and potentially hepatic stellate cells $(\mathrm{HSC})^{[15]}$. He et al.$^{[16]}$ demonstrated that CHI3L1 binds to interleukin-13 receptor $\alpha_{2}$ (IL-13R $\alpha 2$ ), activates MAPK (macrophage mitogen-activated protein kinase), protein kinase B/AKT, and Wnt/ $\beta$-catenin signaling, and regulates TGF- $\beta 1$ production via IL-13R 2 2-dependent mechanisms. CHI3L1 also promotes HSC activation and proliferation ${ }^{[4]}$.

Here, we present a water tank model [Figure 2] to explain the relationship between the progression or regression of liver fibrosis and the concentration and increasing speed of CHI3L1. The inlet of water represents the parameters of $\mathrm{CHI} 3 \mathrm{~L} 1$, and the girth of the inlet pipe represents the absolute concentration of 
CHI3L1. The water pressure (inlet water speed) represents the speed of the increase of CHI3L1 concentration in liver. The outlet represents the natural ability of the liver to repair the fibrosis damage (e.g., degradation of the extracellular matrix). The height of the water tank represents the degree (stages) of liver fibrosis. For example, if the water intake is greater than the water outflow, then the height of the water tank (degree of the fibrosis) would increase after a period of time, thus representing a model of chronic liver fibrosis similar to that observed in chronic HBV patients. If treatment, such as antiviral treatment of HBV, was initiated, the water intake would decrease (measured by a reduction in CHI3L1 concentration). Thus, over time, the height of the water tank (degree of fibrosis) would decrease due to natural recovery properties of the liver.

\section{DECLARATIONS}

\section{Authors' contributions}

Drafted the manuscript: Lin B

Edited and approved the manuscript: Wu S, Liu Y, Liu L, Saadiya M

\section{Availability of data and materials}

Not applicable.

\section{Financial support and sponsorship}

None.

\section{Conflicts of interest}

The author declares that there are no conflicts of interest.

\section{Ethical approval and consent to participate}

Not applicable.

\section{Consent for publication}

Not applicable.

\section{Copyright}

(c) The Author(s) 2018.

\section{REFERENCES}

1. Pellicoro A, Ramachandran P, Iredale JP, Fallowfield JA. Liver fibrosis and repair: immune regulation of wound healing in a solid organ. Nat Rev Immunol 2014;14:181-94.

2. Liao B, Wang Z, Lin S, Xu Y, Yi J, Xu M, Huang Z, Zhou Y, Zhang F, Hou J. Significant fibrosis is not rare in Chinese chronic hepatitis B patients with persistent normal ALT. PLoS One 2013;8:e78672.

3. Xu J, Wang QX, Jiang D, Yang LM, Zhao YL, Chen HS, Wei L. Relationship between the genotypes of hepatitis B virus and the severity of liver diseases. Zhonghua Gan Zang Bing Za Zhi 2003;11:11-3.

4. Tao H, Yang JJ, Shi KH, Huang C, Zhang L, Lv XW, Li J. The significance of YKL-40 protein in liver fibrosis. Inflamm Res 2014;63:249-54.

5. Schuppan D. Liver fibrosis: Common mechanisms and antifibrotic therapies. Clin Res Hepatol Gastroenterol 2015;39 Suppl 1:S51-9.

6. Kumagai E, Mano Y, Yoshio S, Shoji H, Sugiyama M, Korenaga M, Ishida T, Arai T, Itokawa N, Atsukawa M, Hyogo H, Chayama K, Ohashi T, Ito K, Yoneda M, Kawaguchi T, Torimura T, Nozaki Y, Watanabe S, Mizokami M, Kanto T. Serum YKL-40 as a marker of liver fibrosis in patients with non-alcoholic fatty liver disease. Sci Rep 2016;6:35282.

7. Kamal SM, Turner B, He Q, Rasenack J, Bianchi L, Al Tawil A, Nooman A, Massoud M, Koziel MJ, Afdhal NH. Progression of fibrosis in hepatitis C with and without schistosomiasis: correlation with serum markers of fibrosis. Hepatology 2006;43:771-9.

8. Zheng M, Cai WM, Zhao JK, Zhu SM, Liu RH. Determination of serum levels of YKL-40 and hyaluronic acid in patients with hepatic fibrosis due to schistosomiasis japonica and appraisal of their clinical value. Acta Trop 2005;96:148-52.

9. Nunes D, Fleming C, Offner G, Craven D, Fix O, Heeren T, Koziel MJ, Graham C, Tumilty S, Skolnik P, Stuver S, Horsburgh CR, Jr., Cotton D. Noninvasive markers of liver fibrosis are highly predictive of liver-related death in a cohort of HCV-infected individuals with 
and without HIV infection. Am J Gastroenterol 2010;105:1346-53.

10. Saitou Y, Shiraki K, Yamanaka Y, Yamaguchi Y, Kawakita T, Yamamoto N, Sugimoto K, Murata K, Nakano T. Noninvasive estimation of liver fibrosis and response to interferon therapy by a serum fibrogenesis marker, YKL-40, in patients with HCV-associated liver disease. World J Gastroenterol 2005;11:476-81.

11. Huang H, Wu T, Mao J, Fang Y, Zhang J, Wu L, Zheng S, Lin B, Pan H. CHI3L1 is a liver-enriched, noninvasive biomarker that can be used to stage and diagnose substantial hepatic fibrosis. OMICS 2015;19:339-45.

12. Fontana RJ, Dienstag JL, Bonkovsky HL, Sterling RK, Naishadham D, Goodman ZD, Lok AS, Wright EC, Su GL, Group H-CT. Serum fibrosis markers are associated with liver disease progression in non-responder patients with chronic hepatitis C. Gut 2010;59:1401-9.

13. Wang L, Liu T, Zhou J, You H, Jia J. Changes in serum chitinase 3-like 1 levels correlate with changes in liver fibrosis measured by two established quantitative methods in chronic hepatitis B patients following antiviral therapy. Hepatol Res 2018;48:E283-29.

14. Pungpapong S, Nunes DP, Krishna M, Nakhleh R, Chambers K, Ghabril M, Dickson RC, Hughes CB, Steers J, Nguyen JH, Keaveny AP. Serum fibrosis markers can predict rapid fibrosis progression after liver transplantation for hepatitis C. Liver Transpl 2008;14:1294-302.

15. Johansen JS, Christoffersen P, Moller S, Price PA, Henriksen JH, Garbarsch C, Bendtsen F. Serum YKL-40 is increased in patients with hepatic fibrosis. J Hepatol 2000;32:911-20.

16. He CH, Lee CG, Dela Cruz CS, Lee CM, Zhou Y, Ahangari F, Ma B, Herzog EL, Rosenberg SA, Li Y, Nour AM, Parikh CR, Schmidt I, Modis Y, Cantley L, Elias JA. Chitinase 3-like 1 regulates cellular and tissue responses via IL-13 receptor alpha2. Cell Rep 2013;4:830-41. 